\title{
Design aesthetic perspective of knitting clothing color study
}

\author{
Liya Wang ${ }^{1, a}$ \\ ${ }^{1}$ Jiangxi Institute of Fashion Technology, Jiangxi, Nanchang, 330201 \\ a78865425@qq.com
}

Keywords: Design aesthetic; Knitting clothing; Color; The article

\begin{abstract}
In knitting clothing design process, need from the view of design aesthetics, the nationality of knitting clothing design and design aesthetic, aesthetic and practical features. Actively explore knitting clothing color design in the design of aesthetic subject and object, etc by revelation, color design and human ergonomics and aesthetics and literary theory, combining to create and innovation knitting clothing color design categories and pattern, at the same time to strengthen the promotion of independent knitting clothing brand.
\end{abstract}

\section{The introduction}

The colour of clothing design is very important, it vision plays an important role to human body, so you need to strengthen the garment design in colour on the cognitive and aesthetic feelings. For knitting clothing design, although it is different from general pure art creation, but also in the form of aesthetic and aesthetic temperament and interest as a prerequisite.

\section{Color design and aesthetic characteristics}

Based on the color design aesthetic and practical. For fashion design, it not only has the property of material things, and at the same time also has the properties of spirit, in our country in the construction of the socialist construction of spiritual culture and material culture played an important role. Clothing design aesthetic if lack of practicability, and is only pay attention to the feeling on the vision, will lose the meaning of the clothing itself, so the most important characteristics of clothing design aesthetic is practical in the first place.

Protective function. So-called color protection is through the use of color to prevent any danger to human body health. According to the survey of the relevant knowledge, light-colored clothing weak radiation by the atomic bomb and the dark clothing is heavier, so the general chemical protective clothing will make it white, and with this in mind.

Visual identification. Visual identification is common to have safety and relief as its purpose, to highlight its visibility or make it more eye-catching. For example, in the design of the swimsuit cuhk will use warm color to move, this is because the color of the water towards the blue, green, swimwear is designed to warm color to move can complement each other with water cold tonal color, more eye-catching. In the event of drowning, can also convenient to rescuers found need rescue personnel in a timely manner.

Psychological adjustment. The so-called psychological adjustment refers to the use of the heart is affected by the color, choose the appropriate color to create a special colour atmosphere, make the person's psychology can achieve some degree of emotion regulation ability. General household to take, for example, are mostly used in the design is relatively subdued color, in order to create a relaxed and comfortable home atmosphere, at the same time also can relieve tension, make the person feel carefree.

Depending on the fault. Depending on the fault refers to the people impression of the actual colour with itself exists difference. Such as dark clothing to wear after can let a person look more slender; Colour lightness and purity, the higher the costumes will expand feeling, let the body appear fat some, this phenomenon is known as wrong. In the design, the use of the characteristics of the visual can effectively modify certain physiological deficiency on the human body.

Formal beauty knitting color is mixed. The so-called form refers to according to the form of art and natural life, according to the law to form certain laws and characteristics of beauty. Costume 
design expressive in itself make it pay more attention to the formal beauty. At the same time, along with the development of The Times, people's aesthetic standards are also changing, but the formal beauty of clothing design is still the external performance. For knitting clothing, the rules of formal beauty is six, only in the process of design and the collocation of color pays attention to the aesthetic field of vision, to ensure the unity and coordination.

Balance. Knitting clothing color and hue, but also related to the purity and lightness. Generally speaking, in the process of the combination of the color, dark and warm colors will make integral heavy clothing color and should not have too big area of application. For some of the color and cool color with tall lightness to relatively appears lightsome, can use large area, use can be placed in the upper position. This principle in spring and summer fashion conference Titel2013 fully reflected, as is shown in figure 1.
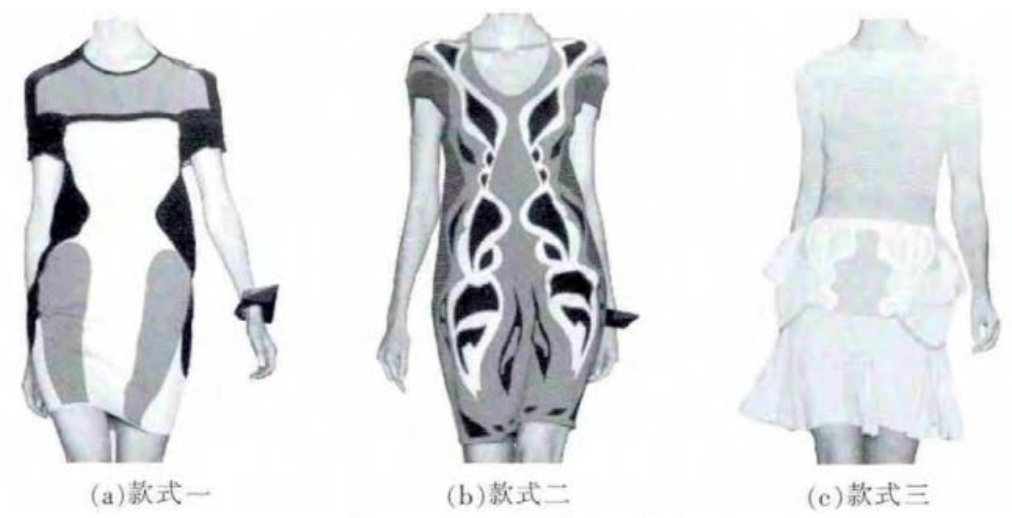

Figure 1 Titel2013 in spring and summer fashion show part of the design

The proportion. In the design of color, it is necessary to timely grasp the color segmentation ratio, the ratio of common relationship with and without rules, ramp rate and golden section proportion. Golden, this kind of follow the golden ratio in the form of a composition is very harmonious, generally on the clothing are embodied in the proportion of 8 or 3:5. The gradient ratio, is to make the color according to the established rules of tiered drive, from one color to another color effect. Its application in knitting clothing design can be done through the combination of density and organization structure. No rules, dare to break the routine on the colour collocation, to seek stimulation and trend of novelty. Rhythm, refers to the lightness and purity in the clothing and so on according to certain way of change over and over again. Color in some free occurs, the line of sight of people will produce a sense of rhythm, the key is to repeat. Stress refers to in order to make clothing no longer appear too drab and on the premise of ensure the color balance in color. Accent color generally can choose a few is proportional to the mass-tone attune of color or high purity color, use area shoulds not be too large, so as to form the visual focus better, at the same time put the visual center on the central location. After using fancy yarn for knitting clothing, sweater would be stronger, such as the use of flash yarn can highlight its metallic luster after feeling, the effect will be better. Echo, echo of so-called emphasis to colorific harmony, for clothing, general won't appear the independence of large-area colour, so tie-in dress will consider the relationship between the shape of the color and texture, grasp the overall scale, to ensure that a unified diversity.

Colour is affected by the national character. Because the different country and nation, nationality in design has become the important characteristics of aesthetic. At present, a lot of knitting clothing designer will be national style into design theme. For example, Chinese style style emphasizes on gold, red and yellow and blue, green, black, etc. The use of colors. In recent years, with the popularity of Indian wind, green, orange and orange colour also gradually popular. Most eastern clothing will make an end of the dark, and vivid color, to ensure the effect of unity and harmony, the west in the clothes in comparison with more gorgeous and lively color.

\section{Color design of aesthetic subject and object of enlightenment}

The main body of the aesthetic design analysis. In the process of people's aesthetic, can give full play to the rational psychological activities such as association, imagination and feelings. 
Emotions. Aesthetic subject emotional because of shock and stimulation caused by the aesthetic object, therefore in the heart of the aesthetic process under dynamic emotion gradually transformed into active emotion. Transformation mainly displays in: consciousness of aesthetic subject can choose emotional aesthetic object. Aesthetic subject has the ability of generalization, the emotion, the emotion and compare their own life experiences, willing to accept the feelings will be strengthened to oneself, this makes the emotion harvest more satisfaction.

Aesthetic value. For fashion design, it itself has aesthetic attributes, and is also the aesthetic psychology of human being materialized. For aesthetic value, it is serving the people, and its practical complement each other, costume design combined with the human body is needed to realize its value, it is also a core design beauty. Clothing to a certain extent, reflects the people's living conditions, need to fully consider the influence of social factors, such as, to make it better service to humans. The choice of clothes is very important, choose the right clothes improve has excellent effect on people's emotions. Modern humans work and life pressure, need timely adjust mood, should choose a few color more partial neutral or no color clothes. People living in the country, in order to make life more colorful, can choose appropriate some relatively bright clothes.

The object of aesthetic design. Clothing design products is the object of aesthetics, the category of it determines people's aesthetic cognition to fashion design products. In the part of consumers and designers in the process of product design, though it is affected by different degree of personality factors, but will still be according to the laws of the public generally follow the color and environment, color and individual achieve perfect harmony. First of all, to ensure the harmonious color itself, there is more than two and the collocation of color is harmonious, at the same time in the process of colour collocation also need to make full use of color to maintain colour such as the rhythm of the beauty and rhythm beauty of harmony. Secondly, considering the colour and the harmony of the human body, generally need to consider from a person's skin color and form and color, because the color is divided into light and dark, so will have in shaping the body results depending on the fault effect, effective use of color arrangement and segmentation is needed to ensure that with the combination of the human body, achieve beautification body of the beauty of harmony between human body and color. In the end, but also to the harmony of color and personality, different cultural level and professional choice of color will be different. People in the process of social, depending on each other's clothing color selection to determine each other's culture and identity. Urban white-collar workers, for example, tend to wear a shirt, it will make people feel more professional attitude.

\section{Conclusion}

Of knitting clothing color design under the design aesthetic vision to carry on the analysis, can not only improve people's perception of aesthetic qualities, as well as the laws of the knitting clothing colour and revealed the essence, easy to enhance people's clothing aesthetic idea, at the same time also can let the designers pay more attention to the quality of the clothes. Organic will knitting clothing color design and human ergonomics and aesthetics, etc, can create a new thinking mode of the our country knitting clothing color design, to facilitate the promotion and development of China's independent brand knitting.

\section{Reference}

[1] Shen Lei; Liu Mengying; Ming-ming jiang; Connie. Design under the aesthetic view of knitting clothing color analysis [J]. Journal of the knitting industry. 2014 (6) : 56-58.

[2] Shen Lei; Connie. Look from missoni brand knitting clothing colour aesthetic [J].. 2007 (01) : 37-39.

[3] guo-min zhou. Analyses the function of manual craft in knitting clothing [J]. Journal of textile industry and technology. 2014 (5) : 77-79.

[4] ming-ming jiang. Knitted garment color design based on replication system research [D]. Southern Yangtze university, 2014 (10). 